\title{
Comprehensive evaluation of low-carbon city in China using intuitionistic fuzzy set theory
}

\author{
Lei Wen ${ }^{1,2}$, Qian Gao ${ }^{1, *}$ \\ ${ }^{1}$ Department of Economics and Management, North China Electric Power University, Baoding, Hebei 071003, China \\ ${ }^{2}$ The Academy of Baoding Low-Carbon Development, Baoding, Hebei 071003, China
}

\section{Email address:}

Weilei0312@sohu.com(Lei Wen),gaoqianbd@163.com(Qian Gao)

\section{To cite this article:}

Lei Wen, Qian Gao. Comprehensive Evaluation of Low-Carbon City in China Using Intuitionistic Fuzzy Set Theory. International Journal of Environmental Protection and Policy. Vol. 1, No. 4, 2013, pp. 68-75. doi: 10.11648/j.ijepp.20130104.14

\begin{abstract}
Low carbon city is the direction of the development of city in the future. Firstly, this paper constructed the comprehensive evaluation index system of the low carbon economy city that including six primary indexes and 12 secondary indexes by summarizing the recent research of low-carbon connotation and low-carbon evaluation index. These indexes are low-carbon economy, low-carbon energy, low-carbon construction, low-carbon traffic, low-carbon society and low-carbon policy. And then, we discussed the development of low-carbon economy of Beijing, Shanghai and Baoding by using intuitionistic fuzzy set approach for urban low-carbon economy comprehensive evaluation index system. The result that Beijing first and Shanghai second and the last is Baoding, that have important practical significance to regulate and guide the construction of low-carbon cities.
\end{abstract}

Keywords: Evaluation of Low-Carbon City, Intuitionistic Fuzzy Sets, Fuzzy Evaluation Method

\section{Introduction}

Energy consumption and emissions of $\mathrm{CO}_{2}$ were increasing rapidly, and these seriously threaten to the global climate. More countries must consider that how to develop a low-carbon economy and realize the transformation of economic development. In 2009, China committed to reducing its carbon dioxide intensity $\left(\mathrm{CO}_{2}\right.$ /unit of gross domestic product, GDP) by $40-45 \%$ by 2020 from a 2005 baseline. In August 2010, after receiving permission from the State Council, the National Development and Reform Commission (NDRC) of China established a Low-Carbon City policy and announced the selection of 5 provinces and 8 cities to pilot the low carbon development work (NDRC, 2010). The five provinces are: Guangdong, Liaoning, Hubei, Shanxi and Yunnan: and the 8 cities are Chongqing, Shenzhen, Xiamen, Tianjin, Hangzhou, Guiyang, Baoding and Nanchang. Until now, over one hundred Chinese cities have been devoted to the construction of low-carbon city that will contribute to the sustainability goals. Therefore, we need to evaluation to a low carbon economy development ability objectively, and provide the recommendation for scientific decision.

Low-carbon city is a new development pattern as well as a development idea (Dai YX and Liu ZL, 2009). The low carbon idea should be penetrated into all fields of the urban development including economic development, energy consumption, construction field, transportation field and social living. The evaluation of low-carbon city is a complicated process; its indicators of the choice not only conform to general indicators selection principle, and also comply with the requirements of low-carbon economy.

The current low-carbon evaluation systems are mainly focused on the studies of the low-carbon economy. For example, in the field of the low-carbon economy evaluation, based on defining what the low-carbon economy is, Zhuang et al. (2011) put forward an index system to measure outputs, consumptions, resources and policies of the low-carbon economy. From the perspective of the low-carbon economy's connotation and its industry chain path, Hu. et al. (2010) proposed a low-carbon economy evaluation index system. From the level of the economic development, the technological development and available resources, Ye. Y and Huang. M (2011) invented nine indicators of the low-carbon economic development evaluation system, and then used the comprehensive analysis and synthesis, obtains the corresponding evaluation results. Xiao-yan Li (2010) Chooses 30 indicators from six aspects of economic development system, then uses fuzzy analytical hierarchy process to evaluate the provincial low carbon economy. 
This paper is organized to determine the evaluation indicators which should be built from seven aspects. They are low-carbon economics, low-carbon energy, low-carbon construction, low-carbon transportation, low-carbon society, and low-carbon policies respectively. In this paper, the intuitionistic fuzzy set theory has been proved to have the ability to evaluate the level of low-carbon city.

The remainder of the paper is organized as follows: section 2 describes the methodologies about the intuitionistic fuzzy set theory in comprehensive evaluation of low-carbon city. Section 3 introduces the calculation of index in the evaluation system. Section 4 presents the comparative result of different cities under low-carbon evaluation index. At last, key findings and recommendations are summarized in Section 5.

\section{Methodologies}

\subsection{Constructing Evaluation Index System}

The evaluation indexes of low-carbon city are not only influenced by the development of economic level, but also by society, energy, transportation and policy and so on. In the design of indicators, it is necessary to consider many factors to meet all needs of the low-carbon development, but the key point is still the economic and energy. The paper follows the basic principles of choosing indicators, such as scientific, systematic operational, dynamic, policy-relevant principles, and forms the target layer, the rule layer and the index layer (Table 1) of the low-carbon economy evaluation index system.

Table 1. The comprehensive evaluation of low-carbon city

\begin{tabular}{|c|c|c|c|}
\hline The target layer & The rule layer & The index layer & unit \\
\hline \multirow{12}{*}{$\begin{array}{l}\text { The comprehensive } \\
\text { evaluation of } \\
\text { low-carbon city }\end{array}$} & \multirow{3}{*}{ Low-carbon economic } & Carbon productivity & Yuan/ton \\
\hline & & Energy intensity & Tons of standard coal/ \\
\hline & & $\begin{array}{l}\text { Non-fossil fuels rate of primary energy } \\
\text { consumption }\end{array}$ & $\%$ \\
\hline & \multirow[t]{2}{*}{ Low-carbon energy } & Intensity of carbon energy & Ton/ yuan \\
\hline & & Elasticity of energy consumption & $\%$ \\
\hline & Low-carbon construction & $\begin{array}{l}\text { Energy consumption per unit area in public } \\
\text { building } \\
\text { Energy consumption per unit area in } \\
\text { residential building }\end{array}$ & $\begin{array}{l}\text { Tons of standard coal } / \mathrm{m}^{2} \\
\text { Tons of standard coal } / \mathrm{m}^{2}\end{array}$ \\
\hline & \multirow[b]{2}{*}{ Low-carbon transportation } & The volume of public transport passenger & $\%$ \\
\hline & & $\begin{array}{l}\text { The proportion of clean energy public } \\
\text { transportation }\end{array}$ & $\%$ \\
\hline & \multirow{3}{*}{ Low-carbon society } & The index of air pollution & $\%$ \\
\hline & & & \\
\hline & & Carbon emissions per capita & Ton/ person \\
\hline & Low-carbon policy & The urban management system & \\
\hline
\end{tabular}

\subsection{The Intuitionistic Fuzzy Sets TOPSIS Method}

Zadeh (1965) proposed fuzzy set theory, then fuzzy theory has been widely used to study fuzzy decision problem. In 1989, Atanassov (1989) expanded the fuzzy sets, put forward the concept of intuitionistic fuzzy sets, compared with the fuzzy set, which gives the degree of membership and non-membership about the element relative to the set $\mathrm{A}$, and also has a strong ability to express uncertain information. In this paper, we combined intuitionistic fuzzy sets with TOPSIS multi-attribute decision making to resolve the problem of supplier selection.

The intuitionistic fuzzy set $A$ in a finite set $X$ can be written as:

$$
A=\left\{<x, \mu_{A}(x), v_{A}(x)>\mid x \in X\right\}
$$

where $\mu_{A}(x), v_{A}(x): X \rightarrow[0,1]$ are membership function and non-membership function,

$$
0 \leq \mu_{A}(x)+v_{A}(x) \leq 1
$$

Define $\pi_{A}=1-\mu_{A}(x)-v_{A}(x)$

as hesitation degree.

$$
\begin{aligned}
& \alpha+\beta=\left\{\mu_{A}(x)+\mu_{B}(x)-\mu_{A}(x) \mu_{B}(x), v_{A}(x) v_{B}(x)\right\} \\
& \alpha \otimes \beta=\left\{\mu_{A}(x) \mu_{B}(x), v_{a}(x)+v_{\beta}(x), v_{A}(x) v_{B}(x) \mid x \in X\right\}
\end{aligned}
$$

$$
\lambda \alpha=\left(1-\left(1-\mu_{A}(x)\right)^{\lambda}, v_{A}(x)^{\lambda}\right)
$$

Model and calculation steps:

Set $A=\left\{A_{1}, A_{2}, \ldots A_{n}\right\}$ is a series of being evaluated 
object, $X=\left\{X_{1}, X_{2}, \ldots, X_{n}\right\}$ is a series of evaluate indexes for being evaluated object.

Step 1. Determine the weights of decision makers.

Let $\mathrm{D}_{\mathrm{k}}=\left\{\mu_{k}, \nu_{k}, \pi_{k}\right\}$ be an intuitionistic fuzzy number for rating of $\mathrm{k}_{\mathrm{th}}$ decision maker. Then the weight of $\mathrm{kth}$ decision maker can be obtained as:

$$
\begin{aligned}
\lambda_{k}= & \frac{\left(\mu_{k}+\pi_{k}\left(\frac{\mu_{k}}{\mu_{k}+v_{k}}\right)\right)}{\sum_{k=1}^{l} \pi_{k}\left(\frac{\mu_{k}}{\mu_{k}+v_{k}}\right)} \\
& c_{i j}=\left[1-\prod_{k=1}^{l}\left(1-\mu_{i j}^{(k)}\right)^{\lambda_{k}}, \prod_{k=1}^{l}\left(v_{i j}^{(k)}\right)^{\lambda_{k}}, \prod_{k=1}^{l}\left(1-\mu_{i j}^{(k)}\right)^{\lambda_{k}}-\prod_{k=1}^{l}\left(v_{i j}^{(k)}\right)^{\lambda_{k}}\right] \\
& C=\left[\left(\mu\left(x_{1}\right), v\left(x_{1}\right), \pi\left(x_{1}\right)\right)\left(\mu\left(x_{2}\right), v\left(x_{2}\right), \pi\left(x_{2}\right)\right) n\left(\mu\left(x_{n}\right), v\left(x_{n}\right), \pi\left(x_{n}\right)\right)\right] \\
= & {\left[c_{1}, c_{2}, n, c_{n}\right] }
\end{aligned}
$$

Step 2. Construct Level indicators' aggregated intuitionistic fuzzy decision matrix.

i Build two indicators' aggregate intuitionistic fuzzy decision vector according to the views of decision makers.

Set $C^{(k)}=\left(c_{i j}{ }^{(k)}\right)_{m \times n}$ is each decision maker's intuitionistic fuzzy decision matrix, $\lambda=\left\{\lambda_{1}, \lambda_{2}, \ldots, \lambda_{i}\right\}$

is each decision maker's weight.

ii Determine the weight of two indicators the criteria are calculated by:

Set $q_{j}^{(k)}=\left[\mu_{j}^{(k)}, v_{j}^{(k)}, \pi_{j}^{(k)}\right]$ is the $\mathrm{k}_{\mathrm{th}}$ decision maker's intuitionistic fuzzy number about index, then the weights of

$$
\begin{aligned}
& q_{j}=I F W A_{\lambda}\left(q_{j}^{(1)}, q_{j}^{(2)}, \ldots, q_{j}^{(l)}\right) \\
& =\lambda_{1} q_{j}^{(1)} \oplus \lambda_{2} q_{j}^{(2)} \oplus \lambda_{3} q_{j}^{(3)} \oplus \ldots \oplus \lambda_{l} q_{j}^{(l)} \\
& =\left[1-\prod_{k=1}^{l}\left(1-\mu_{j}^{(k)}\right)^{\lambda_{k}}, \prod_{k=1}^{l}\left(v_{j}^{(k)}\right)^{\lambda_{k}}, \prod_{k=1}^{l}\left(1-\mu_{j}^{(k)}\right)^{\lambda_{k}}-\prod_{k=1}^{l}\left(v_{j}^{(k)}\right)^{\lambda_{k}}\right] \\
& Q=\left[q_{1}, q_{2}, q_{3}, \ldots, q_{j}\right]
\end{aligned}
$$

iii Construction of weighted summary intuitionistic fuzzy decision matrix

$$
\begin{gathered}
C \otimes Q=\left\{\left(x, \mu_{q}(x), v(x)+v_{q}(x)-v(x) \cdot v(x) \cdot v_{q}(x)\right) \mid x \in X\right\} \\
\pi_{q}(x)=1-v(x)-v_{q}(x)-\mu(x) \cdot \mu_{q}(x)+v(x) \cdot v_{q}(x)
\end{gathered}
$$

$C^{\prime}=\left[\left(\mu_{q}\left(x_{1}\right), v_{q}\left(x_{1}\right), \pi_{q}\left(x_{1}\right)\right)\left(\mu_{q}\left(x_{2}\right), v_{q}\left(x_{2}\right), \pi_{q}\left(x_{2}\right)\right) \ldots\left(\mu_{q}\left(x_{n}\right), v_{q}\left(x_{n}\right), \pi_{q}\left(x_{n}\right)\right)\right]$

$=\left[c_{1}^{\prime}, c_{2}^{\prime}, \ldots c_{j}^{\prime}\right]$

where $\quad c_{j}^{\prime}=\left(\mu_{j}^{\prime}, v_{j}^{\prime}, \pi_{j}^{\prime}\right)=\left(\mu_{q}\left(x_{j}\right), v_{q}\left(x_{j}\right), \pi_{q}\left(x_{j}\right)\right)$

iv The comparison with intuitionistic fuzzy decision $S_{w}^{p}(A, C)=1-\frac{1}{\sqrt[p]{n}} \sqrt[p]{\sum_{1}^{n}\left(\frac{1}{3} \varphi_{1}(i)+\frac{1}{3} \varphi_{1}(i)+\frac{1}{3} \varphi_{1}(i)\right)^{p}}$ vector and the index level of similarity

Let $A=\left\{x_{i}, \mu_{a}\left(x_{i}\right), v_{a}\left(x_{i}\right)\right\}$ is the intuitionistic fuzzy set of index grade.

Where $m_{a}(i)=\frac{1}{2}\left(\mu_{a}\left(x_{i}\right)+1-v_{a}\left(x_{i}\right)\right)$ 


$$
\begin{aligned}
& \begin{array}{c}
m_{q}(i)=\frac{1}{2}\left(\mu_{q}\left(x_{i}\right)+1-v_{q}\left(x_{i}\right)\right) \\
\phi_{1}=\left|\mu_{q}\left(x_{i}\right)-\mu_{a}\left(x_{i}\right)\right| \\
\phi_{2}(i)=\left|m_{q}\left(x_{i}\right)-m_{a}\left(x_{i}\right)\right| \\
\phi_{3}(i)=\left|\left(1-v_{a}\left(x_{i}\right)\right)-\left(1-v_{q}\left(x_{i}\right)\right)\right|
\end{array} \\
& \begin{array}{c}
m_{q}(i)=\frac{1}{2}\left(\mu_{q}\left(x_{i}\right)+1-v_{q}\left(x_{i}\right)\right) \\
\phi_{1}=\left|\mu_{q}\left(x_{i}\right)-\mu_{a}\left(x_{i}\right)\right| \\
\phi_{2}(i)=\left|m_{q}\left(x_{i}\right)-m_{a}\left(x_{i}\right)\right| \\
\phi_{3}(i)=\left|\left(1-v_{a}\left(x_{i}\right)\right)-\left(1-v_{q}\left(x_{i}\right)\right)\right|
\end{array} \\
& \begin{array}{c}
m_{q}(i)=\frac{1}{2}\left(\mu_{q}\left(x_{i}\right)+1-v_{q}\left(x_{i}\right)\right) \\
\phi_{1}=\left|\mu_{q}\left(x_{i}\right)-\mu_{a}\left(x_{i}\right)\right| \\
\phi_{2}(i)=\left|m_{q}\left(x_{i}\right)-m_{a}\left(x_{i}\right)\right| \\
\phi_{3}(i)=\left|\left(1-v_{a}\left(x_{i}\right)\right)-\left(1-v_{q}\left(x_{i}\right)\right)\right|
\end{array} \\
& \begin{array}{c}
m_{q}(i)=\frac{1}{2}\left(\mu_{q}\left(x_{i}\right)+1-v_{q}\left(x_{i}\right)\right) \\
\phi_{1}=\left|\mu_{q}\left(x_{i}\right)-\mu_{a}\left(x_{i}\right)\right| \\
\phi_{2}(i)=\left|m_{q}\left(x_{i}\right)-m_{a}\left(x_{i}\right)\right| \\
\phi_{3}(i)=\left|\left(1-v_{a}\left(x_{i}\right)\right)-\left(1-v_{q}\left(x_{i}\right)\right)\right|
\end{array} \\
& w_{j}=\left[1-\prod_{k=1}^{l}\left(1-\mu_{j}^{(k)}\right)^{\lambda_{k}}, \prod_{k=1}^{l}\left(v_{j}^{(k)}\right) \lambda_{k}, \prod_{k=1}^{l}\left(1-\mu_{j}^{(k)}\right) \lambda_{k}-\prod_{k=1}^{l}\left(v_{j}^{(k)}\right)^{\lambda_{k}}\right] \\
& r_{i j}^{\prime}=\left\{\left(\mu_{A_{i}}(x) \cdot \mu_{w}(x), v_{A_{i}}(x)+v_{w}(x)-v_{A_{i}}(x) \cdot v_{w}(x),\right.\right. \\
& \left.\left.1-\mu_{A_{i}}(x) \cdot \mu_{w}(x)-v_{A_{i}}(x)-v_{w}(x)+v_{A_{i}}(x) \cdot v_{w}(x)\right) \mid x \in X\right\}
\end{aligned}
$$

$r_{i j}^{\prime}$ is an element of the aggregated weighted intuitionistic

$$
\begin{array}{cc}
A^{*}=\left(\mu_{A^{*} w}\left(x_{j}\right), v_{A^{*} w}\left(x_{j}\right)\right) & A^{-}=\left(\mu_{A^{-} w}\left(x_{j}\right), n_{A^{-} w}\left(x_{j}\right)\right) \\
\mu_{A^{*} w}\left(x_{j}\right)=\max _{i} \mu_{A_{i} w}\left(x_{j}\right) \quad \mu_{A^{-} w}\left(x_{j}\right)=\min _{i} \mu_{A_{i} w}\left(x_{j}\right) \\
v_{A^{*} w}=\min _{i} v_{A w}\left(x_{j}\right) \quad v_{A^{-} w}\left(x_{j}\right)=\max _{i} v_{A_{i} w}\left(x_{j}\right)
\end{array}
$$

Step 6. Calculate the separation measures.

$$
\begin{aligned}
& S_{i+}=\sqrt{\frac{1}{2 n} \sum_{j=1}^{n}\left(\left(\mu_{A_{1} w}\left(x_{j}\right)-\mu_{A^{*} w}\left(x_{j}\right)\right)^{2}+\left(v_{A_{1} W}\left(x_{j}\right)-v_{A^{*}{ }^{*}}\left(x_{j}\right)\right)^{2}+\left(\pi_{A_{1} w}\left(x_{j}\right)-v_{A^{*} w}\left(x_{j}\right)\right)^{2}\right)} \\
& S_{i-}=\sqrt{\frac{1}{2 n} \sum_{j=1}^{n}\left(\left(\mu_{A_{1} W}\left(x_{j}\right)-\mu_{A^{-} w}\left(x_{j}\right)\right)^{2}+\left(v_{A_{1} w}\left(x_{j}\right)-v_{A^{-} w}\left(x_{j}\right)\right)^{2}+\left(\pi_{A_{1} w}\left(x_{j}\right)-v_{A^{*} w}\left(x_{j}\right)\right)^{2}\right)}
\end{aligned}
$$

Step 7. Calculate the relative closeness coefficient.

$$
K_{i}=\frac{S_{i-}}{S_{i+}+S_{i-}}
$$

After the relative closeness coefficient of each alternative is determined, alternatives are ranked according to descending order $\mathrm{K}_{\mathrm{i}}$.

\section{Data Collection and Calculation Processing}

In this paper, the three cities including Beijing, Shanghai, Baoding as the evaluation objects to verify practicality and reliability of the comprehensive evaluation index system of low-carbon city. Beijing, as the capital, its economic and politics have strong representation in China with rapid development and expedition. The Chongming Dongtan Eco-city in Shanghai was designed from urban planning, ecological development, sustainable energy, waste management, green building, and transportation planning. It is likely to became the first carbon-neutral area in the word, where an ecosystem characterized by low carbon, water saving and energy saving will generated $(\mathrm{Fu} \mathrm{Y}$, Wang, 2008). Baoding has put forward the plan of "Electricity Valley of China". Six industrial systems including optic-electricity, wind power, electricity savings, electricity storage, trans-electricity, and power automation have been formed in recent years (Yuan XL, Zhong YY, 2010). So the three cities are choosing as typical cities to process the calculation of low-carbon index and analyze for low-carbon development.

Data in this paper are from China Statistical Yearbook, 
China Energy Statistical Yearbook, and Yearbook of China Transportation and Communications, and statistical literature that are compiled by National Bureau of Statistics and Bureau of Energy. The data are collected from the year 2011.

Suppose the three cities are represented by $A_{1}, A_{2}, A_{3}$, three decision makers $\mathrm{DM}_{1}, \mathrm{DM}_{2}$ and $\mathrm{DM}_{3}$. The calculation steps are as follows:

Step 1. Determine the weights of decision makers.

The importance language describe and weights of three experts are shown in Table 4

Table 2. Classification of decision-makers's importance

\begin{tabular}{lccc}
\hline & DM1 & DM2 & DM3 \\
\hline $\begin{array}{l}\text { Linguistic } \\
\text { terms }\end{array}$ & Very important & medium & important \\
Weights & 0.406 & 0.238 & 0.356 \\
\hline
\end{tabular}

Table 3. Linguistic terms description of decision-makers and indicators

\begin{tabular}{ll}
\hline Linguistic terms & Intuitionistic fuzzy set \\
\hline Very important(VI) & $(0.90,0.10)$ \\
Important(I) & $(0.75,0.20)$ \\
Medium important(M) & $(0.50,0.45)$ \\
Unimportant(U) & $(0.35,0.60)$ \\
Very unimportant(VU) & $(0.10,0.90)$ \\
\hline
\end{tabular}

According to the Eq (4) calculate the weight of decision-makers as follows:

$$
\begin{aligned}
& \lambda_{D M 1}=\frac{0.9}{0.9+\left(0.75+0.05 \frac{0.75}{0.95}\right)+\left(0.50+0.05 \frac{0.50}{0.95}\right)}=0.406 \\
& \lambda_{D M 2}=\frac{\left(0.50+0.05 \frac{0.50}{0.95}\right)}{0.9+\left(0.50+0.05 \frac{0.50}{0.95}\right)+\left(0.75+0.05 \frac{0.75}{0.95}\right)}=0.238 \\
& \lambda_{D M 3}=\frac{\left(0.75+0.05 \frac{0.75}{0.95}\right)}{0.9+\left(0.50+0.05 \frac{0.50}{0.95}\right)+\left(0.75+0.05 \frac{0.75}{0.95}\right)}=0.356
\end{aligned}
$$

Step 2. Construct Level indicators' aggregated intuitionistic fuzzy decision matrix.

i Build two indicators' aggregate intuitionistic fuzzy decision vector according to the views of decision makers.

Table 4. Linguistic terms description of devide index level

\begin{tabular}{|c|c|c|c|c|c|c|c|c|c|}
\hline Supplier & & $\mathbf{A}_{1}$ & & & $\mathbf{A}_{2}$ & & & $\mathbf{A}_{3}$ & \\
\hline \multirow{2}{*}{ Criteria } & $\mathrm{D}$ & $\mathrm{D}$ & $\mathrm{D}$ & $\mathrm{D}$ & $\mathrm{D}$ & $\mathrm{D}$ & $\mathrm{D}$ & $\mathrm{D}$ & $\mathrm{D}$ \\
\hline & $\mathrm{M}_{1}$ & $\mathrm{M}_{2}$ & $\mathrm{M}_{3}$ & $\mathrm{M}_{1}$ & $\mathrm{M}_{2}$ & $\mathrm{M}_{3}$ & $\mathrm{M}_{1}$ & $\mathrm{M}_{2}$ & $\mathrm{M}_{3}$ \\
\hline $\mathrm{C}_{1}$ & VG & $\mathrm{G}$ & G & $\mathrm{G}$ & VG & G & $\mathrm{G}$ & $\mathrm{G}$ & M \\
\hline $\mathrm{C}_{2}$ & $\mathrm{G}$ & M & G & VG & $\mathrm{G}$ & G & VG & $\mathrm{G}$ & M \\
\hline $\mathrm{C}_{3}$ & M & G & $\mathrm{G}$ & $\mathrm{G}$ & $\mathrm{G}$ & G & $\mathrm{G}$ & VG & G \\
\hline $\mathrm{C}_{4}$ & G & G & VG & G & M & G & VG & $\mathrm{G}$ & M \\
\hline $\mathrm{C}_{5}$ & M & M & $\mathrm{M}$ & M & G & G & VG & M & G \\
\hline $\mathrm{C}_{6}$ & VG & G & $\mathrm{G}$ & G & G & VG & M & $\mathrm{G}$ & G \\
\hline $\mathrm{C}_{7}$ & $\mathrm{M}$ & $\mathrm{G}$ & VG & M & B & M & G & $\mathrm{G}$ & G \\
\hline $\mathrm{C}_{8}$ & G & VG & $\mathrm{G}$ & M & VG & G & G & VG & G \\
\hline $\mathrm{C}_{9}$ & M & $\mathrm{G}$ & VG & $\mathrm{G}$ & G & VG & G & M & G \\
\hline $\mathrm{C}_{10}$ & M & G & $\mathrm{G}$ & $\mathrm{G}$ & B & $\mathrm{G}$ & G & G & G \\
\hline $\mathrm{C}_{11}$ & G & G & VG & VG & $\mathrm{G}$ & G & M & VG & M \\
\hline $\mathrm{C}_{12}$ & G & M & B & M & M & $\mathrm{G}$ & VG & $\mathrm{G}$ & $\mathrm{G}$ \\
\hline
\end{tabular}

\begin{tabular}{ll}
\hline Linguistic terms & Intuitionistic fuzzy set \\
\hline Very $\operatorname{good}(\mathrm{VG})$ & $(1.00,0.00)$ \\
Good(G) & $(0.75,0.15)$ \\
Medium good(M) & $(0.50,0.40)$ \\
Bad(B) & $(0.25,0.65)$ \\
Very bad(VB) & $(0.10,0.90)$ \\
\hline
\end{tabular}

Table 5. The ratings given by the experts

The aggregated intuitionistic fuzzy decision matrix according to the $\mathrm{Eq}(5)$ :

Table 6. Aggregated intuitionistic fuzzy decision matrix

\begin{tabular}{cccc}
\hline $\mathbf{C}$ & $\mathbf{A}_{\mathbf{1}}$ & $\mathbf{A}_{\mathbf{2}}$ & $\mathbf{A}_{\mathbf{3}}$ \\
\hline $\mathrm{C}_{1}$ & $(1.000,0.000,0.000)$ & $(1.000,0.000,0.000)$ & $(0.680,0.213,0.107)$ \\
$\mathrm{C}_{2}$ & $(0.705,0.189,0.105)$ & $(1.000,0.000,0.000)$ & $(1.000,0.000,0.000)$ \\
$\mathrm{C}_{3}$ & $(0.669,0.223,0.108)$ & $(0.750,0.150,0.100)$ & $(1.000,0.000,0.000)$ \\
$\mathrm{C}_{4}$ & $(1.000,0.000,0.000)$ & $(0.705,0.189,0.105)$ & $(1.000,0.000,0.000)$ \\
$\mathrm{C}_{5}$ & $(0.500,0.400,0.100)$ & $(0.669,0.223,0.108)$ & $(1.000,0.000,0.000)$ \\
$\mathrm{C}_{6}$ & $(1.000,0.000,0.000)$ & $(1.000,0.000,0.000)$ & $(0.669,0.223,0.108)$ \\
$\mathrm{C}_{7}$ & $(1.000,0.000,0.000)$ & $(0.449,0.449,0.102)$ & $(0.750,0.150,0.100)$ \\
$\mathrm{C}_{8}$ & $(1.000,0.000,0.000)$ & $(1.000,0.000,0.000)$ & $(1.000,0.000,0.000)$ \\
$\mathrm{C}_{9}$ & $(1.000,0.000,0.000)$ & $(1.000,0.000,0.000)$ & $(0.705,0.189,0.105)$ \\
$\mathrm{C}_{10}$ & $(0.669,0.223,0.108)$ & $(0.675,0.213,0.112)$ & $(0.750,0.150,0.100)$ \\
$\mathrm{C}_{11}$ & $(1.000,0.000,0.000)$ & $(0.609,0.282,0.109)$ & $(1.000,0.000,0.000)$ \\
$\mathrm{C}_{12}$ & $(0.564,0.319,0.117)$ & $(0.539,0.344,0.117)$ & $(1.000,0.000,0.000)$ \\
\hline
\end{tabular}

ii Determine the weight of two indicators

Table 7. The evaluation of criteria's importance

\begin{tabular}{cccc}
\hline \multirow{2}{*}{ Criteria } & \multicolumn{3}{c}{ Decision maker } \\
\cline { 2 - 4 } & DM $_{\mathbf{1}}$ & DM $_{\mathbf{2}}$ & DM $_{3}$ \\
\hline $\mathrm{C}_{1}$ & $\mathrm{VI}$ & $\mathrm{VI}$ & $\mathrm{I}$ \\
$\mathrm{C}_{2}$ & $\mathrm{VI}$ & $\mathrm{I}$ & $\mathrm{I}$ \\
$\mathrm{C}_{3}$ & $\mathrm{I}$ & $\mathrm{M}$ & $\mathrm{I}$ \\
$\mathrm{C}_{4}$ & $\mathrm{I}$ & $\mathrm{VI}$ & $\mathrm{I}$ \\
$\mathrm{C}_{5}$ & $\mathrm{I}$ & $\mathrm{I}$ & $\mathrm{I}$ \\
$\mathrm{C}_{6}$ & $\mathrm{VI}$ & $\mathrm{I}$ & $\mathrm{VI}$ \\
$\mathrm{C}_{7}$ & $\mathrm{I}$ & $\mathrm{VI}$ & $\mathrm{VI}$ \\
$\mathrm{C}_{8}$ & $\mathrm{VI}$ & $\mathrm{VI}$ & $\mathrm{M}$ \\
$\mathrm{C}_{9}$ & $\mathrm{VI}$ & $\mathrm{M}$ & $\mathrm{I}$ \\
$\mathrm{C}_{10}$ & $\mathrm{I}$ & $\mathrm{VI}$ & $\mathrm{I}$ \\
$\mathrm{C}_{11}$ & $\mathrm{M}$ & $\mathrm{M}$ & $\mathrm{M}$ \\
$\mathrm{C}_{12}$ & $\mathrm{M}$ & $\mathrm{I}$ & $\mathrm{M}$ \\
\hline
\end{tabular}




$Q=\left(\begin{array}{l}0.861,0.128,0.011 \\ 0.828,0.151,0.021 \\ 0.705,0.243,0.052 \\ 0.799,0.170,0.031 \\ 0.750,0.200,0.050 \\ 0.876,0.118,0.006 \\ 0.855,0.133,0.013 \\ 0.823,0.171,0.007 \\ 0.797,0.183,0.020 \\ 0.799,0.170,0.031 \\ 0.500,0.450,0.050 \\ 0.576,0.371,0.053\end{array}\right)^{T}$

iii Construction of weighted summary intuitionistic fuzzy decision matrix

Table 8. Summary intuitionistic fuzzy decision matrix

\begin{tabular}{|c|c|c|c|}
\hline $\mathbf{C}$ & $\mathbf{A}_{1}$ & $\mathbf{A}_{2}$ & $\mathbf{A}_{3}$ \\
\hline $\mathrm{C}_{1}$ & $(0.861,0.128,0.011)$ & $(0.861,0.128,0.011)$ & $(0.586,0.314,0.101)$ \\
\hline $\mathrm{C}_{2}$ & $(0.584,0.311,0.105)$ & $(0.828,0.151,0.021)$ & $(0.828,0.151,0.021)$ \\
\hline $\mathrm{C}_{3}$ & $(0.472,0.411,0.117)$ & $(0.529,0.356,0.115)$ & $(0.705,0.243,0.052)$ \\
\hline $\mathrm{C}_{4}$ & $(0.799,0.170,0.031)$ & $(0.563,0.327,0.110)$ & $(0.799,0.170,0.031)$ \\
\hline $\mathrm{C}_{5}$ & $(0.375,0.520,0.105)$ & $(0.502,0.378,0.120)$ & $(0.750,0.200,0.050)$ \\
\hline $\mathrm{C}_{6}$ & $(0.876,0.118,0.006)$ & $(0.876,0.118,0.006)$ & $(0.586,0.315,0.100)$ \\
\hline $\mathrm{C}_{7}$ & $(0.855,0.133,0.013)$ & $(0.384,0.522,0.094)$ & $(0.641,0.263,0.096)$ \\
\hline $\mathrm{C}_{8}$ & $(0.823,0.171,0.007)$ & $(0.823,0.171,0.007)$ & $(0.823,0.171,0.007)$ \\
\hline $\mathrm{C}_{9}$ & $(0.797,0.183,0.020)$ & $(0.797,0.183,0.020)$ & $(0.562,0.337,0.101)$ \\
\hline $\mathrm{C}_{10}$ & $(0.535,0.355,0.111)$ & $(0.539,0.346,0.114)$ & $(0.599,0.294,0.107)$ \\
\hline $\mathrm{C}_{11}$ & $(0.500,0.450,0.050)$ & $(0.305,0.605,0.090)$ & $(0.500,0.450,0.050)$ \\
\hline $\mathrm{C}_{12}$ & $(0.325,0.572,0.103)$ & $(0.310,0.587,0.102)$ & $(0.576,0.371,0.053)$ \\
\hline
\end{tabular}

iv The comparison with intuitionistic fuzzy decision vector and the index level of similarity

Table 9. The index level of similarity

\begin{tabular}{ccccccc}
\hline Criteria & Supplier & VG & G & M & B & VB \\
\hline & $\mathrm{A}_{1}$ & 0.712 & 0.828 & 0.868 & 0.588 & 0.388 \\
$\mathrm{~B}_{1}$ & $\mathrm{~A}_{2}$ & 0.727 & 0.875 & 0.823 & 0.573 & 0.373 \\
& $\mathrm{~A}_{3}$ & 0.755 & 0.925 & 0.795 & 0.545 & 0.345 \\
& $\mathrm{~A}_{1}$ & 0.748 & 0.862 & 0.740 & 0.552 & 0.352 \\
$\mathrm{~B}_{2}$ & $\mathrm{~A}_{2}$ & 0.675 & 0.816 & 0.819 & 0.626 & 0.426 \\
& $\mathrm{~A}_{3}$ & 0.731 & 0.915 & 0.819 & 0.569 & 0.369 \\
& $\mathrm{~A}_{1}$ & 0.575 & 0.766 & 0.876 & 0.725 & 0.525 \\
& $\mathrm{~A}_{2}$ & 0.529 & 0.720 & 0.827 & 0.771 & 0.571 \\
$\mathrm{~B}_{3}$ & $\mathrm{~A}_{3}$ & 0.598 & 0.798 & 0.939 & 0.702 & 0.502 \\
\hline
\end{tabular}

Get level indicators' evaluation level based on Table 6.
Table 10. Level indicators' evaluation level

\begin{tabular}{cccc}
\hline $\begin{array}{c}\text { Criteria } \\
\text { Supplier }\end{array}$ & $\mathbf{B}_{1}$ & $\mathbf{B}_{2}$ & $\mathbf{B}_{3}$ \\
\hline $\mathrm{A}_{1}$ & $\mathrm{M}$ & $\mathrm{G}$ & $\mathrm{M}$ \\
$\mathrm{A}_{2}$ & $\mathrm{G}$ & $\mathrm{M}$ & $\mathrm{M}$ \\
$\mathrm{A}_{3}$ & $\mathrm{G}$ & $\mathrm{G}$ & $\mathrm{M}$ \\
\hline
\end{tabular}

Table 11. Level indicators' aggregated intuitionistic fuzzy decision matrix

\begin{tabular}{cccc}
\hline Criteria & $\mathbf{A}_{\mathbf{1}}$ & $\mathbf{A}_{\mathbf{2}}$ & $\mathbf{A}_{\mathbf{3}}$ \\
\hline $\mathrm{B}_{1}$ & $(0.500,0.400,0.100)$ & $(0.750,0.150,0.100)$ & $(0.750,0.150,0.100)$ \\
$\mathrm{B}_{2}$ & $(0.750,0.150,0.100)$ & $(0.500,0.400,0.100)$ & $(0.750,0.150,0.100)$ \\
$\mathrm{B}_{3}$ & $(0.500,0.400,0.100)$ & $(0.500,0.400,0.100)$ & $(0.500,0.400,0.100)$ \\
\hline
\end{tabular}

Step 3. Determine the weights of level index.

Table 12. The evaluation of criteria's importance

\begin{tabular}{cccc}
\hline & DM $_{1}$ & DM $_{2}$ & DM $_{3}$ \\
\hline $\mathrm{B}_{1}$ & $\mathrm{VI}$ & $\mathrm{M}$ & $\mathrm{I}$ \\
$\mathrm{B}_{2}$ & $\mathrm{I}$ & $\mathrm{I}$ & $\mathrm{M}$ \\
$\mathrm{B}_{3}$ & $\mathrm{I}$ & $\mathrm{VI}$ & $\mathrm{I}$ \\
\hline
\end{tabular}




$$
w=\left(\begin{array}{l}
(0.797,0.183,0.020) \\
(0.680,0.267,0.053) \\
(0.799,0.170,0.031))
\end{array}\right)^{T}
$$

Step 4. Construct aggregated weighted intuitionistic fuzzy decision matrix.

Table 13. Aggregated weighted intuitionistic fuzzy decision matrix

\begin{tabular}{cccc}
\hline $\mathbf{B}$ & $\mathbf{A}_{\mathbf{1}}$ & $\mathbf{A}_{\mathbf{2}}$ & $\mathbf{A}_{3}$ \\
\hline $\mathrm{B}_{1}$ & $(0.398,0.510,0.092)$ & $(0.598,0.306,0.097)$ & $(0.398,0.510,0.092)$ \\
$\mathrm{B}_{2}$ & $(0.510,0.377,0.113)$ & $(0.340,0.560,0.100)$ & $(0.340,0.560,0.100)$ \\
$\mathrm{B}_{3}$ & $(0.599,0.294,0.107)$ & $(0.599,0.294,0.107)$ & $(0.399,0.502,0.099)$ \\
\hline
\end{tabular}

Step 5. Obtain intuitionistic fuzzy positive-ideal solution and intuitionistic fuzzy negative-ideal solution.

Table 14. Intuitionistic fuzzy positive-ideal solution and negative-ideal solution

\begin{tabular}{cccc}
\hline & $\mathbf{B}_{1}$ & $\mathbf{B}_{2}$ & $\mathbf{B}_{3}$ \\
\hline $\mathrm{A}^{+}$ & $(0.598,0.306,0.097)$ & $(0.510,0.377,0.113)$ & $(0.399,0.502,0.099)$ \\
$\mathrm{A}^{-}$ & $(0.398,0.510,0.092)$ & $(0.340,0.560,0.100)$ & $(0.599,0.294,0.107)$ \\
\hline
\end{tabular}

Step 6. Calculate the separation measures.

Table 15. The separation measures

\begin{tabular}{ccc}
\hline Supplier & $\mathbf{S}^{+}$ & $\mathbf{S}^{-}$ \\
\hline $\mathrm{A}_{1}$ & 0.128 & 0.079 \\
$\mathrm{~A}_{2}$ & 0.121 & 0.09 \\
$\mathrm{~A}_{3}$ & 0.12 & 0.091 \\
\hline
\end{tabular}

Step 7. Calculate the relative closeness coefficient.

Table 16. The relative closeness coefficient

\begin{tabular}{cc}
\hline Supplier & $\mathbf{K}_{\mathbf{i}}$ \\
\hline $\mathrm{A}_{1}$ & 0.381 \\
$\mathrm{~A}_{2}$ & 0.428 \\
$\mathrm{~A}_{3}$ & 0.432 \\
\hline
\end{tabular}

3 partner were ranked according to the relative closeness coefficient of $\mathrm{Ki}$, The alternatives were ranked as $\mathrm{A} 3>\mathrm{A} 2>\mathrm{A} 1$, so $\mathrm{A} 3$ is the best. According to the result above, it is easy to conclude that the low-carbon economy development capability of Beijing is the strongest, Shanghai is stronger than Baoding, and Baoding has the weakest low-carbon economy development capability.

\section{Result and Discussion}

Based on the above calculations, we can easily find that comprehensive indicators based on 6 rule layers and 12 index layers can explain the level of low-carbon development effectively. The comprehensive evaluation system can lead to a relative accurate comparisons and conclusions about whether certain cities or provinces are or are not low-carbon. Beijing ranks first in terms of being low-carbon city, as the nation's capital, with a highly-developed, economically-productive commercial sector. Shanghai to Beijing, China's financial hub, ranks well in terms of low-carbon when the indicator is based on low-carbon energy. Also to Beijing, this low-carbon transportation does not rank well in terms of energy consumption and $\mathrm{CO}_{2}$ emissions. Shanghai also ranks poorly in terms of energy use per capita or per employee for residential and commercial buildings, respectively. Baoding was ranked slightly worse than the other two in terms of comprehensive indexes of low-carbon system. It can be seen that Baoding should make more effort to improve energy efficiency, but also to develop secondary industries with lower carbon intensity.

For the economic sector, the industrial share of regional GDP was used as the denominator, but a better value would be provincial or city industrial sector value added.

For the energy sector, the indicator used is calculated based on total energy demand by province. This approach favors large hydropower producers and exporters such as Hebei province. It accords more closely with supply region for consumption.

For the transport sector, it would be helpful to have more detailed information on usage of all public transportation modes, and the total person trip- kilometers for all private travel in cars and taxis, as well as the total energy consumption of these travel modes in order to develop more detailed indicators and metrics. This information, however, is also not readily available at the provincial and city level for China.

\section{Conclusion}

The results presented above for China illustrate that comprehensive evaluation indicators of low-carbon city based on intuitionistic fuzzy set theory which could explain and reflect the low-carbon development of cities or provinces effectively. This paper constructed the comprehensive evaluation index system of the low carbon economy city that including six primary indexes and 12 secondary indexes by summarizing the research in domestic and foreign of low-carbon connotation and low-carbon evaluation index. These indexes are low-carbon economy, low-carbon energy, low-carbon construction, low-carbon transportation, low-carbon society and low-carbon policy. And then, we discussed the development of low-carbon economy of Beijing, Shanghai and Baoding by using intuitionistic fuzzy set approach for urban low-carbon economy comprehensive evaluation index system. The result that Beijing first and Shanghai second and the last is Baoding, that have important practical significance to regulate and guide the construction of low-carbon cities.

City is an important platform of low-carbon development. 
Many Chinese cities have made their efforts to construct low-carbon city in recent years, among which some establish the overall low-carbon city strategy planning, some construct demonstrative area, some pay attention to specific such as energy, industry, transportation, and some emphasize the scientific research and international cooperation.

There is still a long way to go before achieving the low-carbon development objective. Certain items need to be concerned in the future.

\section{Acknowledgements}

The key projects of central university research in Education Ministry (ID:12ZX21): low-carbon urban construction monitoring and simulation optimization decision system research

\section{References}

[1] Atanassov. K Moreon intuitionistic fuzzy sets[J]. Fuzzy sets and Systems, 1989, 33: 37-46.

[2] Dai YX. A study on low-carbon city development: Concept formation and measurement setting. Mod Urban Res 2009; (11): 7-12.
[3] Fu Y, Wang YL, Li D. The studies on development paths of low-carbon cities. Impact Sci Soc 2008; (2): 5-11.

[4] Hu Dali, Ding Shuai. Small low-carbon economic evaluation index system. Science and Technology Progress and Policy, 2010 (22) :160-164.

[5] Liu ZL, Dai YX, Dong CG, Qi Y. Low-carbon city: Concepts, international practice and implications for China. Urban Stud 2009; 16(6): 1-7, 12.

[6] National Development and Reform Commission (NDRC). (2010). The notice of piloting low-carbon provinces and low-carbon cities. http://www.sdpc.gov.cn/

[7] Xiao-yan Li. An Exploration of Low-carbon Economy of Provinces Based on Fuzzy Analytic Hierarchy Process[J]. East China Economic Management 2010(2), 24-28.zcfb/zcfbtz/2010tz/t20100810_365264.htm

[8] Ye Yichang, Huang Mingfeng. Low-carbon economic developmentindex system and empirical evaluation. Statistics and Decision, 2011 (08):47-49.

[9] Yuan XL, Zhong YY. The practice and system construction of China's low carbon city. Urban Stud 2010; 17(5): 42-7, 8. (in Chinese)

[10] Zadeh. L. A. Fuzzy sets[J]. Inform. And Control. , 1965, 18: 338-356.

[11] Zhuang, G.Y, Pan, J.H, Zhu, S.X. The content of low-carbon economy and comprehensive evaluation index system. Economic Perspectives, 2011 (01):132-136. 